\title{
A KNESER THEOREM FOR VOLTERRA INTEGRAL EQUATIONS
}

\author{
WALTER G. KELLEY
}

ABSTRACT. A connectedness result is obtained for the space of solutions of any one of a class of Volterra integral equations.

1. Introduction. In 1923, Kneser [7] proved for ordinary differential equations that cross sections of the solution funnel emanating from an initial point are compact and connected in some neighborhood of that point. Many interesting generalizations have followed, including that of Hukuhara [4], which showed that the set of solutions of the initial value problem

$$
x^{\prime}=f(t, x), \quad x\left(t_{0}\right)=x_{0}
$$

on a compact interval is a compact and connected subspace of the Banach space of continuous functions on that interval if $f$ is continuous and bounded.

Theorem 3.1 below is a version of this generalized Kneser theorem for Volterra integral equations under very general conditions. The method of proof is based on a connectedness argument given by Hayashi [3] for ordinary differential equations. Other Kneser theorems for integral equations have been proved by Sato [10], [11], Hukuhara [5] and Deimling [1]. In these papers, the authors have assumed that all functions involved in the integral equations are continuous.

As a preliminary result, we have included a convergence theorem for solutions of Volterra integral equations which has less restrictive hypotheses than the similar one given by Miller [9, p. 108].

2. Some preliminary results. We shall be concerned with the Volterra integral equation

$$
x(t)=f(t)+\int_{0}^{t} g(t, s, x(s)) d s,
$$

with hypotheses for $f$ and $g$ similar to those in Chapter II of Miller's book [9].

Received by the editors November $20,1972$.

AMS (MOS) subject classifications (1970). Primary 45D05, 45M99.

Key words and phrases. Volterra integral equations, Kneser theorem, convergence theorem.

(c) American Mathematical Society 1973 
Let $\boldsymbol{R}^{n}$ be $n$-dimensional Euclidean space with norm $|x|$ for $x \in \boldsymbol{R}^{n}$. Let $I$ be a half-open interval of form $[0, c)$, where $0<c \leqq \infty$. Then, in particular, we shall make the following assumptions on $f$ and $g$ :

(H1) $f: I \rightarrow R^{n}$ is continuous,

(H2) $g(t, s, x)$ maps $I \times I \times R^{n}$ into $\boldsymbol{R}^{n}, g$ is measurable in $s$ and $g$ is continuous in $x$ for each fixed pair $(t, s)$,

(H3) for each $b \in I$ and each compact set $B \subset R^{n}$, there is a function $m(t, s)$, measurable in $s$ for fixed $t$, such that $|g(t, s, x)| \leqq m(t, s)$ for $0 \leqq s \leqq t \leqq b, x \in B$ and $\sup \left\{\int_{0}^{t} m(t, s) d s: 0 \leqq t \leqq b\right\}<\infty$,

(H4) for each compact $J \subset I$ and each compact $B \subset R^{n}$, if we let $t \rightarrow t_{0} \in I$, then

$$
\sup \left\{\int_{J}\left|g(t, s, \phi(s))-g\left(t_{0}, s, \phi(s)\right)\right| d s: \phi: J \rightarrow B \text { is continuous }\right\} \rightarrow 0 .
$$

Since equation (E) is of Volterra type, we can assume $g(t, s, x)=0$ if $s>t$. The next lemma will be needed in the proofs of the theorems which follow.

Lemma 2.1. Suppose $g(t, s, x)$ satisfies $(\mathrm{H} 2)-(\mathrm{H} 4)$. Let $J_{1}$ and $J_{2}$ be compact subintervals of $I$ and let $B$ be a compact subset of $\boldsymbol{R}^{n}$. Then for any $\varepsilon>0$, there is a $\delta>0$ such that if $t_{1}, t_{2} \in J_{2}$ and $\left|t_{1}-t_{2}\right|<\delta$, then

$\sup \left\{\int_{J_{1}}\left|g\left(t_{1}, s, \phi(s)\right)-g\left(t_{2}, s, \phi(s)\right)\right| d s: \phi: J_{1} \rightarrow B\right.$

is Lebesgue integrable $\}<\varepsilon$.

Proof. Hypothesis (H4), together with the standard argument that continuity on a compact set implies uniform continuity, yields: for each $\varepsilon>0$, there is a $\delta>0$ such that if $t_{1}, t_{2} \in J_{2}$ and $\left|t_{1}-t_{2}\right|<\delta$, then

$$
\sup \left\{\int_{J_{1}}\left|g\left(t_{1}, s, \phi(s)\right)-g\left(t_{2}, s, \phi(s)\right)\right| d s: \phi: J_{1} \rightarrow B \text { is continuous }\right\}<\varepsilon .
$$

Since the continuous functions mapping $J_{1}$ into $B$ are dense in the Lebesgue-integrable functions mapping $J_{1}$ into $B$, the lemma follows from (H2), (H3) and the Lebesgue dominated convergence theorem. Q.E.D.

As a result of Theorem 1.1 and Corollary 2.6 in [9, Chapter II], we have the following information about equation (E) when $f$ and $g$ satisfy (H1)(H4): there is a $b>0$ and a continuous function $x(t)$ such that $x(t)$ satisfies (E) for $0 \leqq t \leqq b$, and every solution of (E) exists on $[0, b]$. Furthermore, each continuous solution $x(t)$ of $(\mathrm{E})$ can be extended to an interval $[0, \beta)$, where either $\beta=\sup I$ or $\lim \sup _{t \rightarrow \beta^{-}}|x(t)|=+\infty$. 
The next theorem is a generalization of a familiar convergence theorem for ordinary differential equations. (See [2, p. 14].)

THEOREM 2.1. For $k=0,1,2, \cdots$, consider the equations

$$
x(t)=f_{k}(t)+\int_{0}^{t} g_{k}(t, s, x(s)) d s,
$$

where the functions $f_{k}$ and $g_{k}$ satisfy hypotheses (H1)-(H4). Suppose $\lim _{k \rightarrow \infty} f_{k}(t)=f_{0}(t)$ uniformly on compact subintervals of $I$ and

$$
\begin{aligned}
\sup \left\{\int_{0}^{t} \mid g_{k}(t, s, \phi(s))\right. & -g_{0}(t, s, \phi(s)) \mid d s: \\
0 & \leqq t \leqq b, \phi:[0, b] \rightarrow B \text { is continuous }\} \rightarrow 0 \text { as } k \rightarrow \infty
\end{aligned}
$$

for each $b \in I$ and compact $B \subset R^{n}$. Let $x_{k}(t)$ be a solution of $(\mathrm{E})_{k}$ with maximal interval of existence $\left[0, \beta_{k}\right)$ for $k=1,2, \cdots$. Then there is a solution $x_{0}(t)$ of $(\mathrm{E})_{0}$ with maximal interval $[0, \beta)$ and a subsequence $\left\langle x_{k_{i}}\right\rangle_{i=1}^{\infty}$ such that for any $d \in(0, \beta),[0, d] \subset\left[0, \beta_{k_{i}}\right)$ for $i$ sufficiently large and $\lim _{i \rightarrow \infty} x_{k_{i}}(t)=x_{0}(t)$ uniformly on $[0, d]$.

Proof. Let $b_{1}$ and $b_{2}$ belong to $I$ with $b_{1}<b_{2}$, and let $B \subset \boldsymbol{R}^{n}$ be compact. Fix $\varepsilon>0$ and define $K$ to be the compact set

$$
\left\{(t, x) \in\left[0, b_{2}\right] \times \boldsymbol{R}^{n}: \inf _{y \in B}|x-y| \leqq 2 \varepsilon\right\} .
$$

Define $\Phi_{K}$ to be the set of the continuous functions on $\left[0, b_{2}\right]$ with graphs in $K$.

It is easy to see from the convergence assumptions above that the previous lemma implies that a $\delta \in\left(0, b_{2}-b_{1}\right)$ can be chosen so that if $t_{1}$ and $t_{2}$ belong to $\left[0, b_{2}\right]$ with $\left|t_{1}-t_{2}\right|<\delta$, then $\left|f_{k}\left(t_{1}\right)-f_{k}\left(t_{2}\right)\right|<\varepsilon$ and

$$
\sup \left\{\int_{0}^{b_{2}}\left|g_{k}\left(t_{1}, s, \phi(s)\right)-g_{k}\left(t_{2}, s, \phi(s)\right)\right| d s: \phi \in \Phi_{K}\right\}<\varepsilon
$$

for every $k$.

Now let $z(t)$ be a maximally defined solution of $(\mathrm{E})_{k}$ for some $k$ and suppose $(t, z(t)) \in\left[0, b_{1}\right] \times B$ for $t \in\left[0, t_{1}\right]$. Let $t_{2}$ satisfy $0<t_{2}-t_{1}<\delta$. We claim $\left|z\left(t_{1}\right)-z\left(t_{2}\right)\right|<2 \varepsilon$.

If not, there is a smallest $t_{0} \in\left(t_{1}, t_{2}\right]$ such that $\left|z\left(t_{1}\right)-z\left(t_{0}\right)\right|=2 \varepsilon$. Then 
$(t, z(t)) \in K$ for $t \in\left[t_{1}, t_{0}\right]$. Now

$$
\begin{aligned}
\left|z\left(t_{1}\right)-z\left(t_{0}\right)\right| \leqq & \left|f_{k}\left(t_{1}\right)-f_{k}\left(t_{0}\right)\right| \\
& \quad+\left|\int_{0}^{t_{1}} g_{k}\left(t_{1}, s, z(s)\right) d s-\int_{0}^{t_{0}} g_{k}\left(t_{0}, s, z(s)\right) d s\right| \\
< & \varepsilon+\int_{0}^{t_{0}}\left|g_{k}\left(t_{1}, s, z(s)\right)-g_{k}\left(t_{0}, s, z(s)\right)\right| d s .
\end{aligned}
$$

Since the restriction of $z(s)$ to the interval $\left[0, t_{0}\right]$ is a continuous function with graph in $K$, a continuous extension $\phi(s)$ of this function to $\left[0, b_{2}\right]$ can be constructed so that $\phi$ has graph in $K$. We have

$$
\begin{aligned}
\left|z\left(t_{1}\right)-z\left(t_{0}\right)\right|< & \varepsilon+\int_{0}^{t_{0}}\left|g_{k}\left(t_{1}, s, z(s)\right)-g_{k}\left(t_{0}, s, z(s)\right)\right| d s \\
& +\int_{t_{0}}^{b_{2}}\left|g_{k}\left(t_{1}, s, \phi(s)\right)-g_{k}\left(t_{0}, s, \phi(s)\right)\right| d s \\
= & \varepsilon+\int_{0}^{b_{2}}\left|g_{k}\left(t_{1}, s, \phi(s)\right)-g_{k}\left(t_{0}, s, \phi(s)\right)\right| d s<2 \varepsilon .
\end{aligned}
$$

This contradiction implies that $\left|z\left(t_{1}\right)-z\left(t_{2}\right)\right|<2 \varepsilon$.

For $m=1,2, \cdots$, define $\boldsymbol{B}_{m}=\left\{x \in \boldsymbol{R}^{n}:\left|x-f_{0}(0)\right| \leqq m\right\}$ and $J_{m}=$ $\left[0, \alpha_{m}\right]$, where $\left\langle\alpha_{m}\right\rangle_{m=1}^{\infty}$ is a strictly increasing sequence of points in $I$ with $\sup \left\{\alpha_{m}\right\}=\sup I$.

First, consider $J_{1} \times B_{1}$. We can assume without loss of generality that $f_{k}(0) \in B_{1}$ for every $k$. From the above discussion, there is a $\delta_{1} \in\left(0, \alpha_{2}-\alpha_{1}\right)$ so that if $z(t)$ is a maximally defined solution of some $(\mathrm{E})_{k}$ with $(t, z(t)) \in$ $J_{1} \times B_{1}$ for $t \in\left[0, t_{1}\right]$, then $\left|z\left(t_{1}\right)-z(t)\right|<1$ for $t \in\left[t_{1}, t_{1}+\delta_{1}\right]$. In particular, we have that each $x_{k}(t)$ exists on $\left[0, \delta_{1}\right]$ and $\left|x_{k}(t)-x_{k}(0)\right| \leqq 1$ for $t \in$ $\left[0, \delta_{1}\right]$. By the first part of the proof, the family $\left\{x_{k}(t): k=1,2, \cdots\right\}$ is equicontinuous on $\left[0, \delta_{1}\right]$, so the. Ascoli theorem implies that there is a subsequence $\left\langle x_{k_{i}}(t)\right\rangle_{i=1}^{\infty}$ which converges uniformly to a continuous function $x_{0}(t)$ on $\left[0, \delta_{1}\right]$. For each fixed $t \in\left[0, \delta_{1}\right], f_{k_{i}}(t) \rightarrow f_{0}(t)$ and

$$
\int_{0}^{t} g_{k_{i}}\left(t, s, x_{k_{i}}(s)\right) d s \rightarrow \int_{0}^{t} g_{0}\left(t, s, x_{0}(s)\right) d s
$$

as $i \rightarrow \infty$, so $x_{0}(t)$ is a solution of $(\mathrm{E})_{0}$ on $\left[0, \delta_{1}\right]$.

Next, if $\left(\delta_{1}, x_{0}\left(\delta_{1}\right)\right) \in \operatorname{int}\left(J_{1} \times B_{1}\right)$, where $\operatorname{int}\left(J_{1} \times B_{1}\right)$ is the interior of $J_{1} \times B_{1}$, we consider the interval $\left[\delta_{1}, 2 \delta_{1}\right]$ and select a subsequence of $\left\langle x_{k_{i}}\right\rangle$, which we again call $\left\langle x_{k_{i}}\right\rangle$, having a uniform limit on $\left[\delta_{1}, 2 \delta_{1}\right]$. We name this continuous function $x_{0}(t)$ and note that $x_{0}(t)$ is a solution of $(\mathrm{E})_{0}$ on $\left[0,2 \delta_{1}\right]$.

This process is continued until for some integer $j\left(j \delta_{1}, x_{0}\left(j \delta_{1}\right)\right) \notin$ $\operatorname{int}\left(J_{1} \times B_{1}\right)$. Note that $\left(j \delta_{1}, x_{0}\left(j \delta_{1}\right)\right) \in \operatorname{int}\left(J_{2} \times B_{2}\right)$. The subsequence of 
$\left\langle x_{k}(t)\right\rangle_{k=1}^{\infty}$ that we have obtained at this point we call $\left\langle x_{k 1}(t)\right\rangle_{k=1}^{\infty}$, and we define $\tau_{1}=j \delta_{1}$. Also, the sequence $\left\langle x_{k 1}\right\rangle$ converges uniformly to a continuous function $x_{0}(t)$ on $\left[0, \tau_{1}\right]$, and $x_{0}(t)$ is a solution of $(\mathrm{E})_{0}$ on $\left[0, \tau_{1}\right]$.

For $J_{2} \times B_{2}$, we can choose similarly a $\delta_{2} \in\left(0, \alpha_{3}-\alpha_{2}\right)$ and, proceeding as above, obtain a subsequence $\left\langle x_{k 2}\right\rangle$ of $\left\langle x_{k 1}\right\rangle$ and a $\tau_{2}>\tau_{1}$ such that $\lim _{k \rightarrow \infty} x_{k 2}(t)=x_{0}(t)$ uniformly on $\left[0, \tau_{2}\right], x_{0}(t)$ is a solution of $(E)_{0}$ on $\left[0, \tau_{2}\right]$ and $\left(\tau_{2}, x_{0}\left(\tau_{2}\right)\right) \notin \operatorname{int}\left(J_{2} \times B_{2}\right)$.

If this process is repeated for every $J_{m} \times B_{m}$, we obtain finally a sequence $\tau_{1}\left\langle\tau_{2}\left\langle\tau_{3}\left\langle\cdots\right.\right.\right.$ and a nested sequence of subsequences $\left\langle x_{k 1}\right\rangle,\left\langle x_{k 2}\right\rangle, \cdots$ suç that $\lim _{k \rightarrow \infty} x_{k m}(t)=x_{0}(t)$ uniformly on $\left[0, \tau_{m}\right], x_{0}(t)$ is a solution of $(\mathrm{E})_{0}$ on $\left[0, \tau_{m}\right]$ and $\left(\tau_{m}, x_{0}\left(\tau_{m}\right)\right) \notin \operatorname{int}\left(J_{m} \times B_{m}\right)$. Define $\beta=\sup \left\{\tau_{m}\right\}$ and define $\left\langle x_{k_{i}}\right\rangle_{i=1}^{\infty}$ to be the diagonal subsequence $\left\langle x_{k k}\right\rangle_{k=1}^{\infty}$.

Then $x_{0}(t)$ is a solution of $(\mathrm{E})_{0}$ on $[0, \beta)$, and either $\beta=\sup I$ or $\lim \sup _{t \uparrow \beta}\left|x_{0}(t)\right|=+\infty$, so $[0, \beta)$ is the maximal interval of existence for $x_{0}(t)$. The theorem follows immediately. Q.E.D.

COROLlaRY 2.1. Suppose all solutions of $(\mathrm{E})$ exist on a subinterval $[0, d]$ of $I$. Then the family of solutions of $(\mathrm{E})$ on $[0, d]$ is uniformly bounded.

PROof. Let $\left\langle x_{n}\right\rangle_{n=1}^{\infty}$ be a sequence of solutions of (E) with maximal intervals of existence $\left[0, \beta_{n}\right)$. Let $x(t)$ be a solution of (E) given by Theorem 2.1 with maximal interval of existence $[0, \beta)$, and let $\left\langle x_{n_{k}}(t)\right\rangle_{k=1}^{\infty}$ be a subsequence of $\left\langle x_{n}\right\rangle$ such that $\lim _{k \rightarrow \infty} x_{n_{k}}(t)=x(t)$ uniformly on compact subintervals of $[0, \beta)$.

Note that $\beta>d$ since all solutions of (E) exist on $[0, d]$. For $k$ sufficiently large, $\lim _{k \rightarrow \infty} x_{n_{k}}(t)=x(t)$ uniformly on $[0, d]$, and the corollary is established. Q.E.D.

3. A generalized Kneser theorem. For any interval $[a, b]$, we shall let $B[a, b]$ be the metric space of bounded functions from $[a, b]$ into $\boldsymbol{R}^{n}$ with metric $\rho\left(h_{1}, h_{2}\right)=\sup \left\{\left|h_{1}(t)-h_{2}(t)\right|: t \in[a, b]\right\}$ for $h_{1}$ and $h_{2}$ in $B[a, b]$. $C[a, b]$ will denote the subspace of continuous functions on $[a, b]$.

The following theorem is a generalized Kneser theorem for integral equations.

THEOREM 3.1. Suppose all solutions of (E) exist on a subinterval $[0, d]$ of $I$. Then

$$
\Sigma \equiv\{x(t) \in C[0, d]: x(t) \text { is a solution of }(\mathrm{E}) \text { on }[0, d]\}
$$

is compact and connected as a subspace of $C[0, d]$.

Proof. By Corollary 2.1 , there is a number $M>0$ such that $|x(t)| \leqq M$ for every $t \in[0, d]$ and every $x(t) \in \Sigma$. Redefine $g$ as follows:

$$
\begin{aligned}
& g(t, s, x) \equiv g(t, s, M x /|x|) \quad \text { for } t, s \in I, \quad|x|>M, \\
& \equiv g(t, s, x) \quad \text { for } t, s \in I, \quad|x| \leqq M .
\end{aligned}
$$


Then (H2)-(H4) are still satisfied for $g$, and $\Sigma$ is unaffected by the change in $g$. In fact, the compact sets $B$ appearing in (H3), (H4) and Lemma 2.1 can be replaced by $\boldsymbol{R}^{n}$.

As in the proof of Theorem $2.1, \Sigma$ is an equicontinuous family. Since $\Sigma$ is uniformly bounded, it follows from the Ascoli theorem that $\Sigma$ is precompact. By Theorem $2.1, \Sigma$ is closed. Thus $\Sigma$ is compact.

Suppose $\Sigma$ is not connected. Then $\Sigma=A \cup B$, where $A$ and $B$ are compact and nonempty and $d(A, B)=\gamma>0$, where $d$ is the distance function on pairs of sets induced by the metric $\rho$. Define $F(x) \equiv d(x, A)-$ $d(x, B)$ for $x \in B[0, d]$. The function $F$ is continuous, $F(x) \geqq \gamma$ for $x \in B$ and $F(x) \leqq-\gamma$ for $x \in A$.

Let $x_{B}(t) \in B$ and $x_{A}(t) \in A$. For $k=1,2,3, \cdots$, define

$$
\begin{aligned}
& x_{B, k}(t) \equiv f(0) \quad \text { for } 0 \leqq t<t_{k 1} \text {, } \\
& \equiv x_{B}\left(t_{k 1}\right) \quad \text { for } t_{k 1} \leqq t<t_{k 2}, \\
& \equiv x_{B}\left(t_{k, k-1}\right) \text { for } t_{k, k-1} \leqq t \leqq t_{k, k}=d,
\end{aligned}
$$

where $t_{k, j}=j d \mid k$ for $j=1,2, \cdots, k$. Then $\lim _{k \rightarrow \infty} x_{B, k}(t)=x_{B}(t)$ uniformly on $[0, d]$. Let $\alpha_{B, k}(t, s) \equiv g\left(t, s, x_{B}(s)\right)-g\left(t, s, x_{B, k}(s)\right)$ for all $t, s, k$. If $m(t, s)$ is the function given by hypothesis (H3) for $g$ and $d$, then $\left|\alpha_{B, k}(t, s)\right| \leqq 2 m(t, s)$, and so $\lim _{k \rightarrow \infty} \int_{0}^{t}\left|\alpha_{B, k}(t, s)\right| d s=0$ by the Lebesgue dominated convergence theorem for each $t \in[0, d]$.

Define $x_{A, k}(t)$ and $\alpha_{A, k}(t, s)$ in a similar manner. Then define $\alpha_{k}(u, t, s) \equiv$ $(1-u) \alpha_{B, k}(t, s)+u \alpha_{A, k}(t, s)$ for $u \in[0,1]$. Finally, for $0 \leqq u \leqq 1$ and all $k$, define

$$
\begin{aligned}
x_{u, k}(t) \equiv f(0) & \text { for } 0 \leqq t<t_{k 1}, \\
\equiv f\left(t_{k 1}\right)+\int_{0}^{t_{k 1}}\left[g\left(t_{k 1}, s, x_{u, k}(s)\right)+\alpha_{k}\left(u, t_{k 1}, s\right)\right] d s & \text { for } t_{k 1} \leqq t<t_{k 2}, \\
\cdot & \\
\equiv & \cdot \\
\equiv & \\
&
\end{aligned}
$$

Note that $x_{0, k}(t)=x_{B, k}(t)$ and $x_{1, k}(t)=x_{A, k}(t)$ for $t \in[0, d]$.

Consider, for fixed $k$, the function from $[0,1]$ to $B[0, d]$ which maps $u$ to $x_{u, k}(t)$. For $u_{0} \in[0,1]$ and a sequence $\left\langle u_{i}\right\rangle$ of points in $[0,1]$ converging to $u_{0}$, it follows from an induction argument using the dominated 
convergence theorem that $\lim _{i \rightarrow \infty} x_{u_{i}, k}(t)=x_{u_{0}, k}(t)$ uniformly on $[0, d]$. Thus $F\left(x_{u, k}\right)$ is continuous as a function of $u$ for each $k$. There exists an $N$ such that for $k \geqq N, F\left(x_{B, k}\right)>0$ and $F\left(x_{A, k}\right)<0$. Then for each $k \geqq N$, there is a $u_{k} \in[0,1]$ such that $F\left(x_{u_{k}, k}\right)=0$.

For each $k \geqq N$, define

Now

$$
x_{k}(t) \equiv f(t)+\int_{0}^{t}\left[g\left(t, s, x_{u_{k}, k}(s)\right)+\alpha_{k}\left(u_{k}, t, s\right)\right] d s .
$$

$$
\left|x_{k}(t)\right| \leqq \sup _{t \in[0, d]}|f(t)|+\sup _{t \in[0, d]} \int_{0}^{t} 3 m(t, s) d s \text { for } t \in[0, d]
$$

so $\left\langle x_{k}(t)\right\rangle_{k=N}^{\infty}$ is uniformly bounded on $[0, d]$. Let $t_{1}$ and $t_{2}$ be in $[0, d]$ with $t_{1}<t_{2}$. Then

$$
\begin{aligned}
\left|x_{k}\left(t_{1}\right)-x_{k}\left(t_{2}\right)\right| \leqq & \left|f\left(t_{1}\right)-f\left(t_{2}\right)\right| \\
& +\left|\int_{0}^{t_{2}}\left[g\left(t_{1}, s, x_{u_{k}, k}(s)\right)-g\left(t_{2}, s, x_{u_{k}, k}(s)\right)\right] d s\right| \\
& +\left|\int_{0}^{t_{2}}\left[\alpha_{k}\left(u_{k}, t_{1}, s\right)-\alpha_{k}\left(u_{k}, t_{2}, s\right)\right] d s\right| .
\end{aligned}
$$

From Lemma 2.1 and the fact that the functions $x_{A, k}, x_{B, k}$ and $x_{u_{k}, k}$ are step functions on $[0, d]$ for each $k$, the right-hand side of the above inequality tends to zero uniformly in $k$ as $t_{2}-t_{1} \rightarrow 0$. Thus $\left\langle x_{k}(t)\right\rangle_{k=N}^{\infty}$ is equicontinuous. Select a subsequence $\left\langle x_{k_{i}}(t)\right\rangle_{i=1}^{\infty}$ such that $\lim _{i \rightarrow \infty} x_{k_{i}}(t)=$ $x(t)$ uniformly on $[0, d]$.

For each $t \in[0, d]$ and $k \geqq N$, let $j(t, k)$ have the property that $t \in\left[t_{k, j(t, k)}, t_{k, j(t, k)+1}\right)$. Then

$$
\begin{aligned}
\left|x_{k}(t)-x_{u_{k, k}}(t)\right| \leqq & \left|f(t)-f\left(t_{k, j(t, k)}\right)\right| \\
& +\left|\int_{0}^{d}\left[g\left(t, s, x_{u_{k, k}}(s)\right)-g\left(t_{k, j(t, k)}, s, x_{u_{k}, k}(s)\right)\right] d s\right| \\
& +\left|\int_{0}^{d}\left[\alpha_{k}\left(u_{k}, t, s\right)-\alpha_{k}\left(u_{k}, t_{k, j(t, k)}, s\right)\right] d s\right| .
\end{aligned}
$$

Again by Lemma 2.1, the right-hand side tends to zero as $k \rightarrow \infty$ uniformly on $[0, d]$. Thus $x(t)$ is the uniform limit of $\left\langle x_{u_{k_{i}}, k_{i}}(t)\right\rangle_{i=1}^{\infty}$ on $[0, d]$. Hence $F(x)=0$.

From the definition of $x_{k}(t)$ and the fact that $\int_{0}^{t}\left|\alpha_{k}\left(u_{k}, t, s\right)\right| d s \rightarrow 0$ as $k \rightarrow \infty$ for each $t \in[0, d]$, it is easy to see that $x(t) \in \Sigma$. From this contradiction it follows that $\Sigma$ is connected. Q.E.D.

The next corollary follows immediately from the above theorem and the knowledge that there is a $d>0$ such that every solution of (E) exists on $[0, d]$. 
Corollary 3.1. There is a $d>0$ such that, for every $c \in[0, d]$, $\{x(c): x \in \Sigma\}$ is compact and connected in $\boldsymbol{R}^{n}$.

We note that Theorem 3.1 can be proven similarly, and somewhat more easily, for the Volterra equation

$$
x(t)=f(t)+\int_{0}^{t} a(t, s) g(s, x(s)) d s,
$$

with the conditions on the functions $a$ and $g$ given in [8].

\section{BIBLIOGRAPHY}

1. Klaus Deimling, Eigenschaften der Lösungsmenge eines Systems von VolterraIntegralgleichungen, Manuscripta Math. 4 (1971), 201-212. MR 44 \#3087.

2. P. Hartman, Ordinary differential equations, Wiley, New York, 1964. MR 30 $\# 1270$.

3. K. Hayashi, On the solutions of the system of ordinary differential equations, Mem. Coll. Sci. Univ. Kyoto Ser. A Math. 28 (1953), 19-25. MR 15, 623.

4. M. Hukuhara, Sur une généralisation d'un théorème de Kneser, Proc. Japan Acad. 29 (1953), 154-155. MR 15, 623.

5. - Sur l'application qui fait correspondre à une point un continu bicompact, Proc. Japan Acad. 31 (1955), 5-7. MR 16, 1140.

6. E. Kamke, Zur Theorie der Systeme gewöhnlicher Differentialgleichungen. II, Acta. Math. 58 (1932), 57-85.

7. H. Kneser, Über die Lösungen eines systems gewöhnlicher Differentialgleichungen das der Lipschitzschen Bedingung nicht genügt, S.-B. Preuss. Akad. Wiss. Phys.-Math. Kl. (1923), 171-174.

8. R. K. Miller and G. R. Sell, Existence, uniqueness and continuity of solutions of integral equations, Ann. Mat. Pura Appl. (4) 80 (1968), 135-152. MR 40 \#663.

9. R. K. Miller, Nonlinear Volterra integral equations, Benjamin, California, 1971.

10. T. Satō, Sur l'equation intégrale non linéaire de Volterra, Compositio Math. 11 (1953), 271-290. MR 15, 714.

11. - Sur l'application qui fait correspondre à une courbe une famille de courbes, Proc. Japan Acad. 31 (1955), 1-4. MR 16, 1120.

Department of Mathematics, University of Oklahoma, Norman, Oklahoma 73069 